Article

\title{
Multiple Poliovirus Proteins Repress Cytoplasmic RNA Granules
}

\author{
Jonathan D. Dougherty ${ }^{\dagger}$, Wei-Chih Tsai and Richard E. Lloyd * \\ Received: 13 October 2015; Accepted: 17 November 2015; Published: 25 November 2015 \\ Academic Editor: Eric O. Freed \\ Department of Molecular Virology and Microbiology, Baylor College of Medicine, Houston, TX 77030, USA; \\ Wei-Chih.Tsai@bcm.edu \\ * Correspondence: rlloyd@bcm.edu; Tel.: +1-713-798-8993 \\ † Current address; jonathan.dougherty@nih.gov.
}

\begin{abstract}
We have previously shown that poliovirus (PV) infection induces stress granule (SG) formation early in infection and then inhibits the formation of SG and disperses processing bodies (PBs) by the mid-phase of infection. Loss of SG was linked to cleavage of G3BP1 by viral 3C proteinase $\left(3 \mathrm{C}^{\mathrm{pro}}\right)$, however dispersal of PBs was not strongly linked to cleavage of specific factors by viral proteinases, suggesting other viral proteins may play roles in inhibition of SG or PB formation. Here we have screened all viral proteins for roles in inducing or inhibiting the formation of RNA granules by creating fusions with mCherry and expressing them individually in cells. Expression of viral proteins separately revealed that the capsid region P1, $2 \mathrm{~A}^{\mathrm{pro}}, 3 \mathrm{~A}, 3 \mathrm{C}^{\mathrm{pro}}$, the protease precursor $3 \mathrm{CD}$ and $3 \mathrm{D}$ polymerase all affect RNA granules to varying extents, whereas $2 \mathrm{BC}$ does not. 2A $\mathrm{A}^{\text {pro }}$, which cleaves eIF4GI, induced SGs as expected, and entered novel foci containing the SG nucleating protein G3BP1. Of the two forms of G3BP, only G3BP1 is cleaved by a virus proteinase, $3 C^{\text {pro }}$, whereas G3BP2 is not cleaved by $3 C^{\text {pro }}$ or $2 A^{\text {pro }}$. Surprisingly, $3 C D$, which contains proteinase activity, differentially repressed PBs but not SGs. Further, both $2 \mathrm{~A}^{\text {pro }}$ and $3 C^{\text {pro }}$ expression dispersed PBs, however molecular targets were different since PB dispersal due to $2 \mathrm{~A}^{\text {pro }}$ and heat shock protein (Hsp) 90 inhibition but not $3 \mathrm{C}^{\text {pro }}$, could be rescued by application of oxidative stress to cells. The data indicate that PV repression of SGs and PBs is multifactorial, though protease function is dominant.
\end{abstract}

Keywords: stress granule; processing body; P-body; G3BP1; Tia1; poliovirus; Rck/p54

PACS: J0101

\section{Introduction}

Somatic cells form two major classes of cytoplasmic RNA granules known as stress granules (SGs) and processing bodies (PBs) that transiently store silenced messenger ribonucleoproteins (mRNPs). Stress granules form in response to many types of environmental stress and serve as triage centers for silenced mRNPs. As such, they contain stalled translation initiation complexes consisting of small ribosome subunits, mRNA, translation initiation factors and many RNA binding proteins [1-3]. In contrast, PBsare usually constitutively present in cells, however, they also respond to stress with by increasing their size and number. Unlike SGs, PBs contain mRNAs in association with $5^{\prime}$ and $3^{\prime}$-mediated RNA decay factors, mRNA decapping, deadenylation, micro RNA (miRNA)-mediated mRNA silencing, and mRNA storage and are devoid of most translation initiation factors and ribosome subunits $[1,4,5]$. Both SGs and PBs are very dynamic foci, with protein and mRNA components rapidly exchanging with the surrounding cytosol. Stress granules and PBs are distinguished by their functional protein constituents and key marker proteins. For SGs this 
includes initiation factors eIF4G, eIF3, eIF4E, small ribosome subunits and SG-nucleating proteins G3BP and Tia-1, among others. PBs are identified by containing RNA helicase Rck/p54 (also known as DDX6), the 5'-3' exoribonuclease Xrn1, mRNA-decapping enzymes 1a and 2, (Dcp1a and Dcp2), and PABP1-dependent poly(A) nucleases 2 and 3, (Pan2/Pan3) [6].

SGs and PBs function as extensions of translation regulation mechanisms and spatially coordinate networks of mRNPs that are stored or actively translated. This is especially true during non-steady state conditions in the cell. In addition, defects in RNA granule functions have been linked to multiple neurodegenerative, developmental disorders and an increasing role of RNA granules in cancer biology is emerging [7-10]. Because of the prominence of RNA granules in regulation of gene expression, many viruses are known to antagonize RNA granule function to maintain efficient rates of expression of their proteins and to prevent their genomes and transcripts from being compartmentalized in these silencing compartments [11,12].

We have previously shown that poliovirus (PV) disrupts SG formation by cleavage of the key SG nucleating protein G3BP1 and that PV disperses PBs in conjunction with cleavage and degradation of Dcp1a, Pan3 and Xrn1 [13,14]. Prevention of G3BP cleavage restores SG formation in infected cells suggesting this is a major mechanism of SG disruption, however the mechanism of PB disruption is less clear [14,15]. To further characterize mechanisms of RNA granule disruption by poliovirus we have expressed all the major proteins encoded by the virus individually and determined their effect on modulating both SG and PB assembly. We confirm that PV 2A proteinase (2A ${ }^{\text {pro) }}$ expression strongly induces SGs but surprisingly it disperses PBs. 2A pro enters unique cytoplasmic foci containing G3BP1, but does not cleave either G3BP1 or G3BP2 and the 2A-mediated dispersal of PBs could be overcome by exogenous stress in the absence of other viral proteins. In contrast, PB dispersal by $3 C$ proteinase $\left(3 \mathrm{C}^{\text {pro }}\right)$ could not be countered by exogenous stress and uniquely dispersed $\mathrm{PB}$ foci containing ectopic Dcp1a. Other viral polypeptides had little or modest effects on RNA granules, however, the precursor of $3 C^{\text {pro }}, 3 \mathrm{CD}$, surprisingly did not block SG formation but did affect PBs. Overall, the data indicate that $3 C^{\text {pro }}$ is the key viral protein that represses both types of cytoplasmic RNA granules.

\section{Materials and Methods}

\subsection{Cell Culture, Transfections and Infections}

HeLa and A549 cells were used in experiments and grown in Dulbecco's Modified Eagle's Media (DMEM) supplemented with $10 \%$ fetal bovine serum (FBS) and incubated at $37{ }^{\circ} \mathrm{C}$ with $5 \% \mathrm{CO}_{2}$. For transfections cells were plated at a density of $1.5 \times 10^{5}$ cells/well in 12-well plates and $24 \mathrm{~h}$ later cells were transfected with indicated plasmid DNAs using Xtremegene-HP transfection reagent (Roche, Basel, Switzerland) or Lipofectamine 3000 (ThermoFisher, Rockford, IL, USA) according to manufacturer's protocol. Cells were routinely transfected with $0.5 \mu \mathrm{g}$ DNA per $1.5 \times 10^{5}$ cells. Poliovirus Type 1 (Mahoney strain) was used to infect cells as indicated at a multiplicity of infection of 10 .

\subsection{Antibodies}

The following antibodies were used in immunoblots and immunofluorescence: rabbit anti-G3BP1 [14], mouse anti-G3BP1 (Bethyl Labs, Montgomery, TX, USA), rabbit anti-G3BP2 (Assay Biotech, Sunnyvale, CA, USA), goat anti-TIA1 (Santa Cruz, Dallas, TX, USA), goat anti-TIAR (Santa Cruz), anti-Rck/p54(DDX6) (gift from CE Cameron), anti-rabbit Alexa Fluor 488 (ThermoFisher, Rockford, IL, USA), anti-mouse Alexa Fluor 647 (Invitrogen), anti-rabbit Texas Red (ThermoFisher), anti-goat Texas Red (ThermoFisher). 


\subsection{Plasmids and Cloning}

Plasmids encoding poliovirus protein-mCherry fusions were also created using the In-Fusion system. PCR products were generated from the plasmid pTPOV [16] using the following primers:

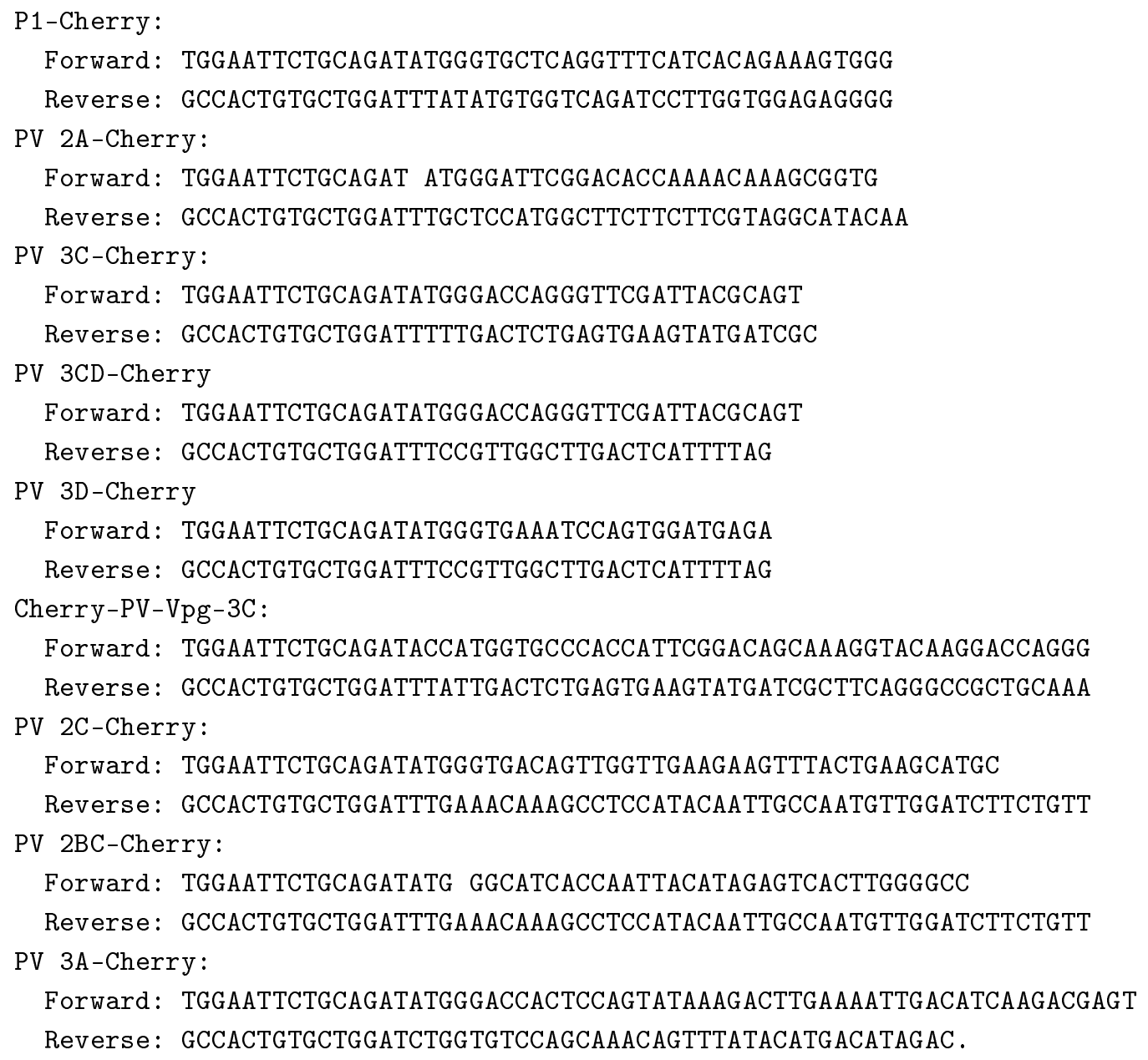

Plasmids PCR amplicons were inserted into vectors pcDNA4/TO-NCherry-mycHisA or pcDNA4/TO-CCherry-mycHisA. These vectors were obtained from T. Albrect and E.J. Wagner (University of Texas Medical Branch, Galveston, TX, USA) and were created from pcDNA4/TO-NCherry-mycHisAby (ThermoFisher) by insertion of mCherry bearing the identical multiple cloning sites in frame upstream or downstream of mCherry.

\subsection{Immunofluorescence}

Cells seeded on glass coverslips and transfected with appropriate plasmids were allowed to express the transgene for $14-24 \mathrm{~h}$, after which the cells were fixed with $4 \%$ paraformaldehyde in PEM (80 mM PIPES, $5 \mathrm{mM}$ EGTA, $2 \mathrm{mM} \mathrm{MgCl} \mathrm{M}_{2}$ ). Cells were then permeabilized in $0.5 \%$ Triton X-100 and blocked with $5 \%$ bovine serum albumin (BSA) in Tris-buffered saline- $0.1 \%$ tween- 20 (TBS-T). The cells were then incubated with the appropriate primary antibodies for two hours at room temperature or overnight at $4{ }^{\circ} \mathrm{C}$. After washing in TBS-T five times, five minutes each, secondary antibodies were applied in TBS-T for $1 \mathrm{~h}$, and then cells were washed with TBS-T five additional times. Cover slips were mounted on microscope slides with Vectashield mounting medium with DAPI (Vector Labs, Burlingame, CA, USA) to visualize nuclei. The coverslips were analyzed by microscopy on a Nikon TE2000 inverted microscope, or deconvolution microscopy was performed with an Applied Precision DeltaVision image restoration microscopy with conservative deconvolution algorithms. For single 
cell quantification of SGs or PBs, images of 50-100 transfected cells per plasmid were scored for RNA granule content using Image $\mathrm{J}$.

\section{Results}

\subsection{PV Proteins Drive Assembly or Disassembly of SGs}

PV infection transiently induces SG early in infection before actively dispersing SG through cleavage of G3BP1 [14]. In order to test the contribution of other PV proteins in stimulating the stress granule response noted during infection, we generated expression constructs tagged with the mCherry fluorophore that encoded all major regions of the complete viral polypeptide, many representing completely processed PV proteins, but also some as precursors. We expressed PV-fusion proteins independently in HeLa cells and after $16 \mathrm{~h}$ expression examined cells for SG by immunofluorescence microscopy. Expression of the mCherry control vector did not significantly induce formation of SGs, as indicated by Tia1 and G3BP1 antibody labeling, nor did expression of the viral P1 capsid region (containing viral protein (VP)1, VP2, VP3 and VP4), 2BC, 3Cpro, 3CD, or 3 Dpolymerase (3Dpol). In contrast, PV 2A ${ }^{\text {pro }}$ was found to strongly induce $S G$ formation (Figure 1). Similar results were found in A549 cells (not shown). The failure of $3 C^{\text {pro }}$ or $3 C D$ to induce SG was not surprising since $3 C^{\text {pro }}$ strongly antagonizes SG formation. The finding that $2 \mathrm{~A}^{\text {pro }}$ induced SGs was not surprising because it mediates the cleavage of host translation factor eIF4G and results in translation shutoff that generates stalled translation initiation complexes that are stored in SGs [17]. The distribution of expressed P1, $3 C D, 3 C^{\text {pro }}$ in cells was diffuse, however, $2 A^{\text {pro }}$ was frequently found in more irregular patchy accumulations that sometimes resembled foci. Examination of foci indicated they contained G3BP1 but not Tia1, thus were not bona fide stress granules (Figure 1C).

The colocalization of G3BP1 with 2A ${ }^{\text {pro }}$ prompted examination of cleavage of G3BP1 and G3BP2 by both PV proteases. Potential cleavage of G3BP2 in PV infection has not been previously examined. G3BP2 is a close homolog of G3BP1 that interacts with G3BP1 and contains very high identity in the amino terminal regions and has been proposed to provide strong SG-nucleating activity and regulate some innate immune functions similar to G3BP1 $[18,19]$. PV 3C ${ }^{\text {pro }}$ substrate specificity requires an AxEQ/G motif in the $\mathrm{P} 4$ through $\mathrm{P} 1^{\prime}$ positions. This motif is present in G3BP1 but the key $\mathrm{P}^{\prime}$ glycine in G3BP1 (Figure 2A, box) is missing in G3BP2. This suggests G3BP2 may be refractory to cleavage at this site, however cleavage at other sites is possible. When lysates from PV-infected cells were probed for G3BP1 and G3BP2 cleavage, only the former was found cleaved (Figure 2). We further probed whether either protease could cleave G3BP1 or G3BP2 in vitro. As expected 3C ${ }^{\text {pro }}$ readily cleaved G3BP1, but G3BP2 was stable when incubated with 3C ${ }^{\text {pro }}$. Additionally, neither G3BP1 nor G3BP2 was cleaved by $2 \mathrm{~A}^{\text {pro }}$. These data underscore the importance of G3BP1 cleavage by poliovirus in controlling SG assembly and raise questions concerning the functional importance of G3BP2 in SG assembly since cleavage of only G3BP1 is sufficient to destroy SG nucleation.

To determine if PV $3 C^{\text {pro }}$ was the only viral protein that blocked SG formation, cells expressing viral polypeptides were stressed with arsenite to induce SG formation and imaged by microscopy (Figure 3A). The number of SG's per cell was determined and, as expected, it was found that expression of $3 \mathrm{C}^{\text {pro }}$ strongly reduced SG formation in response to arsenite. Surprisingly though, $3 C D$, which is the processing precursor of $3 C^{\text {pro }}$, did not inhibit SG formation. $3 C D$ contains efficient proteolytic activity but its substrate specificity is altered from $3 C^{\text {pro }}[20,21]$. In addition, viral proteins $\mathrm{P} 1,2 \mathrm{~A}^{\text {pro }}$ and $3 \mathrm{~A}$ displayed modest but measureable abilities to restrict SG formation when expressed in cells (Figure 3B). This suggested that other viral proteins may aide $3 C^{\text {pro }}$ in restricting the SG assembly responses in cells. 
A
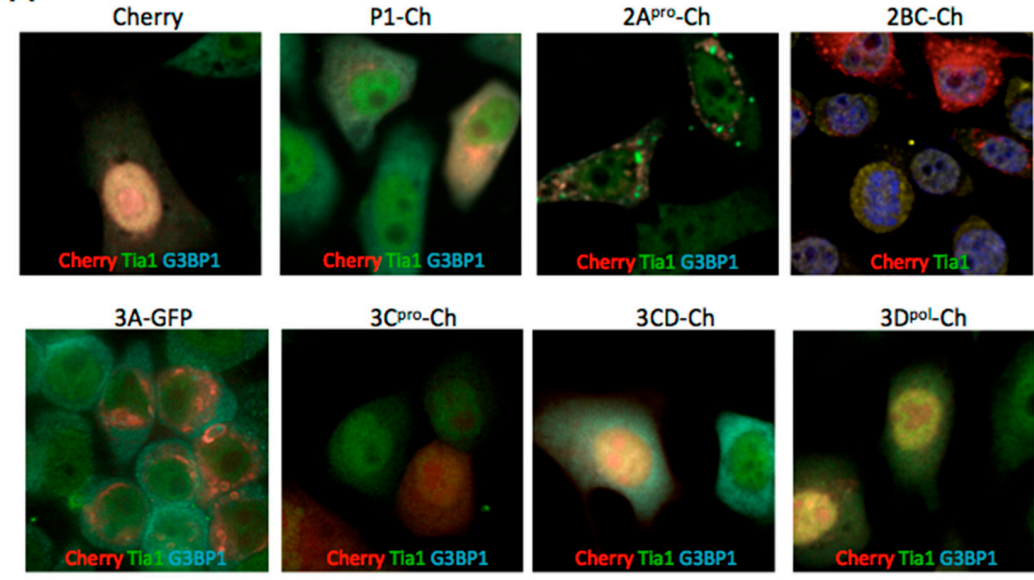

B

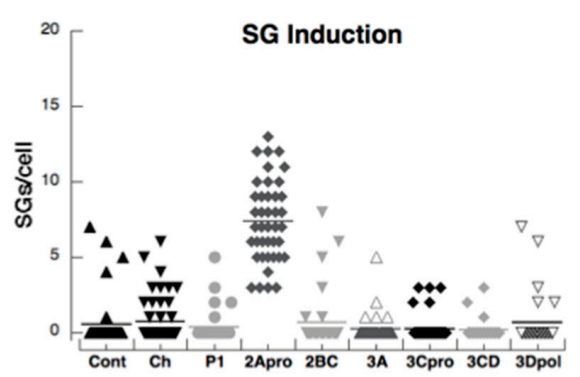

C

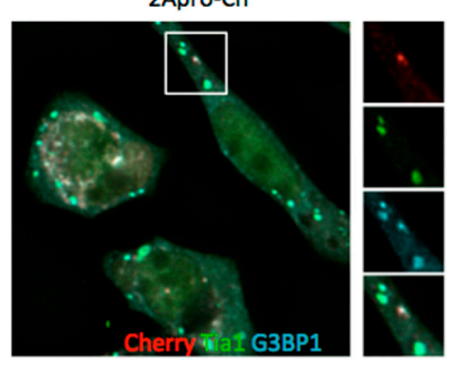

Figure 1. Induction of stress granules (SGs) by expression of poliovirus (PV) proteins. HeLa cells plated on glass coverslips were transfected with expression constructs for mCherry or fusions of mCherry with the indicated viral proteins. PV proteins were expressed in cells for $16 \mathrm{~h}$ before fixation and processing for immunofluorescence (IF) microscopy. (A) Representative images of cells expressing PV fusion proteins. (B) Box plots of the number of stress granules in individual cells expressing mCherry $(\mathrm{Ch})$ or viral proteins with means indicated by horizontal lines. (C) $2 \mathrm{~A}^{\text {pro }}$ appears in unique foci containing G3BP1 but not Tia1.

A

G3BP1 PqRGPRPiReagEQgDiepRRmvRhPDSH G3BP2 PpRGPRPgRgdmEQnDsdnRRiiRyPDSH

B

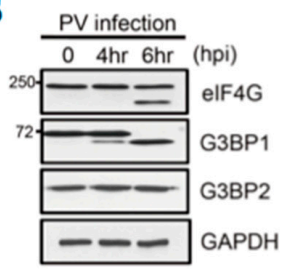

C

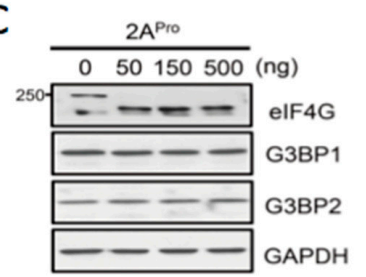

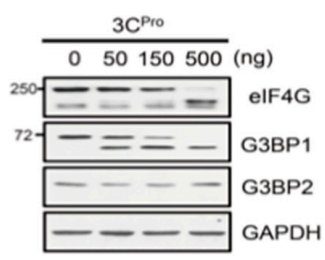

Figure 2. PV infection results in cleavage of G3BP1 but not G3BP2. (A) Sequence alignment of human G3BP1 (amino acids 311-340) and G3BP2 showing the scissile Q-G bond in G3BP1 cleaved by 3Cpro (box) is absent in G3BP2. (B) Immunoblot analysis of G3BP cleavage in PV infection. Cleavage of eIF4G1 is shown as a positive control. (C) In vitro cleavage assays using purified $2 \mathrm{~A}^{\text {pro }}$ and $3 \mathrm{C}^{\text {pro }}$. Proteases were incubated with cell lysate for $60 \mathrm{~min}$ before assay by immunoblot. 
A
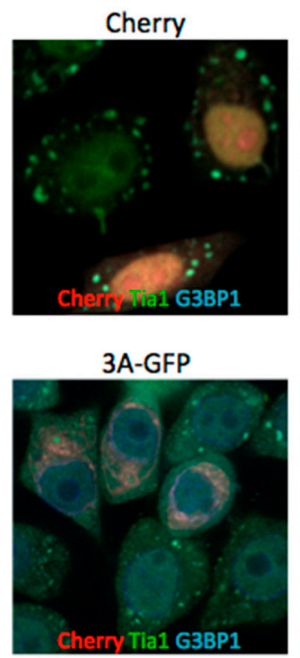

P1-Ch

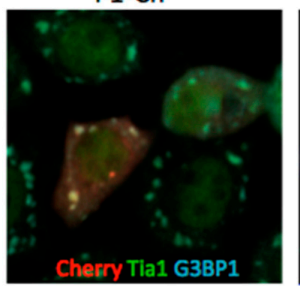

3Cpro-Ch

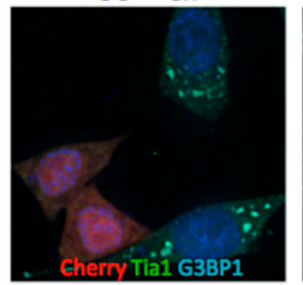

$2 A^{\text {pro-Ch }}$

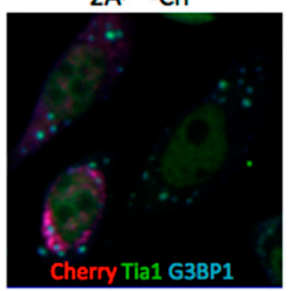

$3 \mathrm{CD}-\mathrm{Ch}$

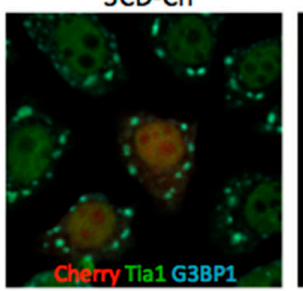

2BC-Ch

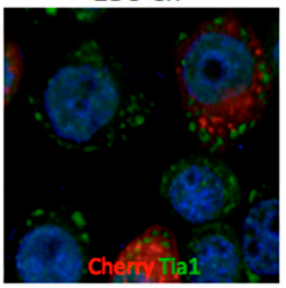

3Dpol-Ch

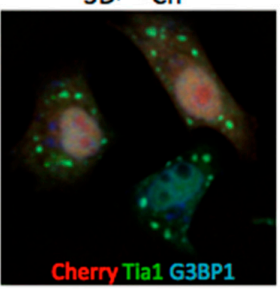

B

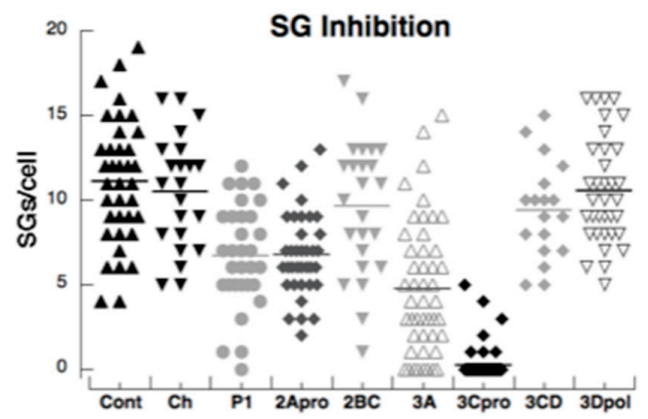

Figure 3. Inhibition of arsenite-induced SGs by expression of PV proteins. (A) Immunofluorescent microscopy of HeLa cells expressing viral proteins for $16 \mathrm{~h}$ and then stressed for 30 min with $200 \mu \mathrm{M}$ sodium arsenite. (B) The number of stress granules/cell in individual cells is expressed in box plots.

\subsection{Both PV Proteases Mediate PB Disruption}

PV or coxsackievirus B3 infection efficiently disperses PBs in mammalian cells by mid-phase of the replicative cycle [13]. We previously linked 3C ${ }^{\text {pro }}$ to cleavage of PB proteins Dcp1a and PAN3, but the spectrum of viral proteins that contribute to $\mathrm{PB}$ dispersion and the mechanisms involved remain unclear. To determine the minimal viral proteins required for disruption of PBs we expressed the panel of viral-Cherry fusion proteins and cells were examined by microscopy for effects on $\mathrm{PB}$ size and abundance. Expression of the mCherry control vector did not significantly alter the size or number of constitutively present PBs, as indicated by antibody labeling of the PB marker protein Rck/p54. Similarly, expression of PV 2BC-Cherry and 3A-Cherry did not result in significant alterations in $\mathrm{PB}$ abundance (Figure 4). Since these proteins are primarily involved in membrane rearrangements that support viral replication, it is not surprising that they did not significantly modulate PBs, which are not membrane bound structures. In contrast, when $2 \mathrm{~A}^{\text {pro }}$-Cherry or $3 C^{\text {pro }}$-Cherry were expressed, endogenous $\mathrm{PBs}$ were significantly dispersed (Figure 4). Since $3 \mathrm{C}^{\text {pro }}$ mediates the cleavage of Dcp1a [13], a PB component involved in translational silencing, mRNA degradation, this was an expected result. However, $2 \mathrm{~A}^{\text {pro }}$-mediated disruption of $\mathrm{PB}$ was surprising, as we have previously determined it does not cleave the three $\mathrm{PB}$ constituents we found that undergo degradation during infection (Xrn1, Dcp1a, or Pan3) [13]. This suggests that PV may target additional components of $\mathrm{PBs}$ or the pathways that control PB formation or maintenance, as $2 \mathrm{~A}$ and $3 \mathrm{C}$ proteinases have distinct substrate specificity. 


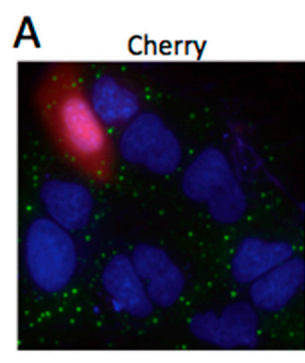

$3 \mathrm{~A}-\mathrm{Ch}$

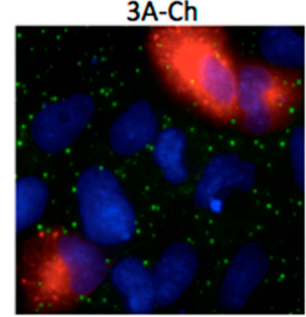

B

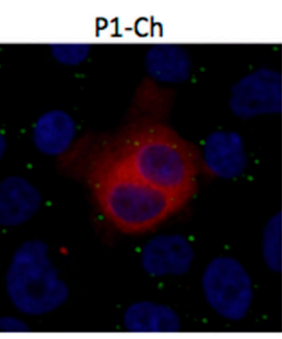

$3 \mathrm{C}^{\text {pro- }} \mathrm{Ch}$

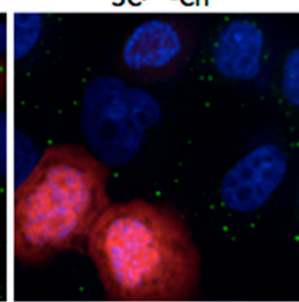

Cherry Rck/p54

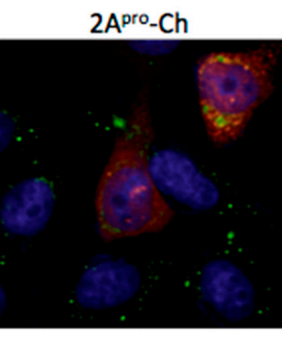

$3 C D-C h$

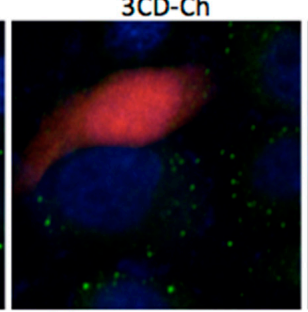

(1)
$2 \mathrm{BC}-\mathrm{Ch}$

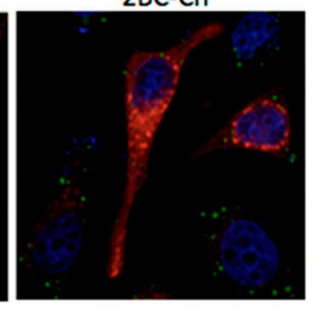

$3 D^{\text {pol- }-C h}$
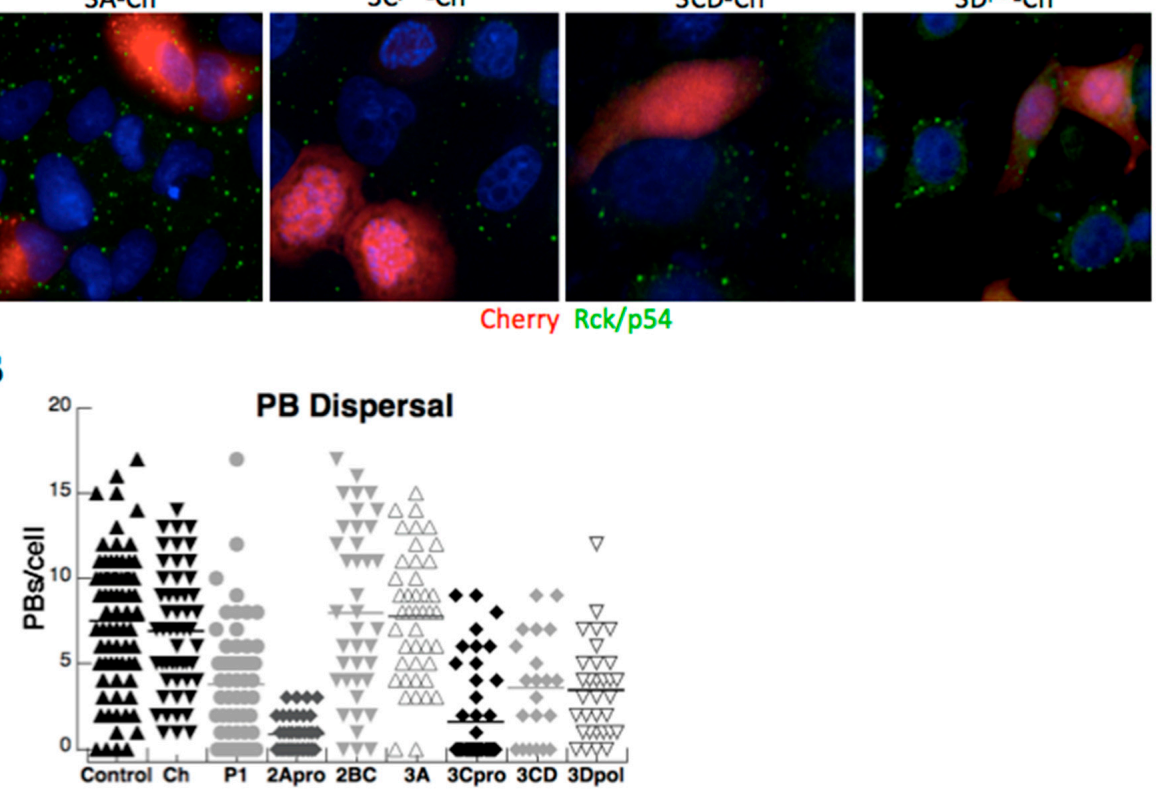

Figure 4. Dispersal of PBs by expression of PV proteins. (A) HeLa cells were transfected with expression constructs encoding mCherry, or PV virus protein fusions as indicated and expression continued for $16 \mathrm{~h}$ before fixation and processing for IF as described previously. Cells were incubated with an anti-Rck/p54 antibody (green) to label endogenous PBs. (B) The number of PBs/cell in individual cells as a measure of PB dispersal is expressed in box plots.

Expression of 3CD modestly reduced the number of PBs in cells, though not as efficiently as $3 C^{\text {pro }}$ itself. This was in contrast to the lack effect of $3 C D$ on SG formation noted above. Surprisingly, expression of the poliovirus polymerase ( $3 \mathrm{D}^{\mathrm{pol}}$ ) had a modest inhibitory effect on $\mathrm{PB}$ formation.

\subsection{Exogenous Stress can Rescue PB Formation following Chemical Dispersal}

Although PBs are constitutively present in cells, application of environmental stress causes PB size and number to increase in cells, likely due to the inhibition of translation and increased number of translationally silenced mRNP complexes [1]. Exogenous stress also induces the formation of SGs, which often form adjacent to PBs. Additionally, the formation of PBs requires the function of the molecular chaperone heat-shock protein 90 (Hsp90). Treatment with geldanamycin (GA), an Hsp90 inhibitor, is able to significantly disperse endogenous PBs [22-24]. In order to investigate the kinetics of PB induction in response to cellular stress and the connections between SGs and PBs, we utilized GA to chemically disperse the endogenous PBs of A549 cells. Subsequently, we added sodium arsenite for thirty minutes to induce oxidative stress. Strikingly, PBs were restored upon stress addition, despite the continued presence of GA at inhibitory concentrations (Figure 5). This suggested that endogenous PBs and stress-induced PBs may be compositionally different, or formed by different mechanisms, as stress-induced PBs do not require Hsp90 function. 

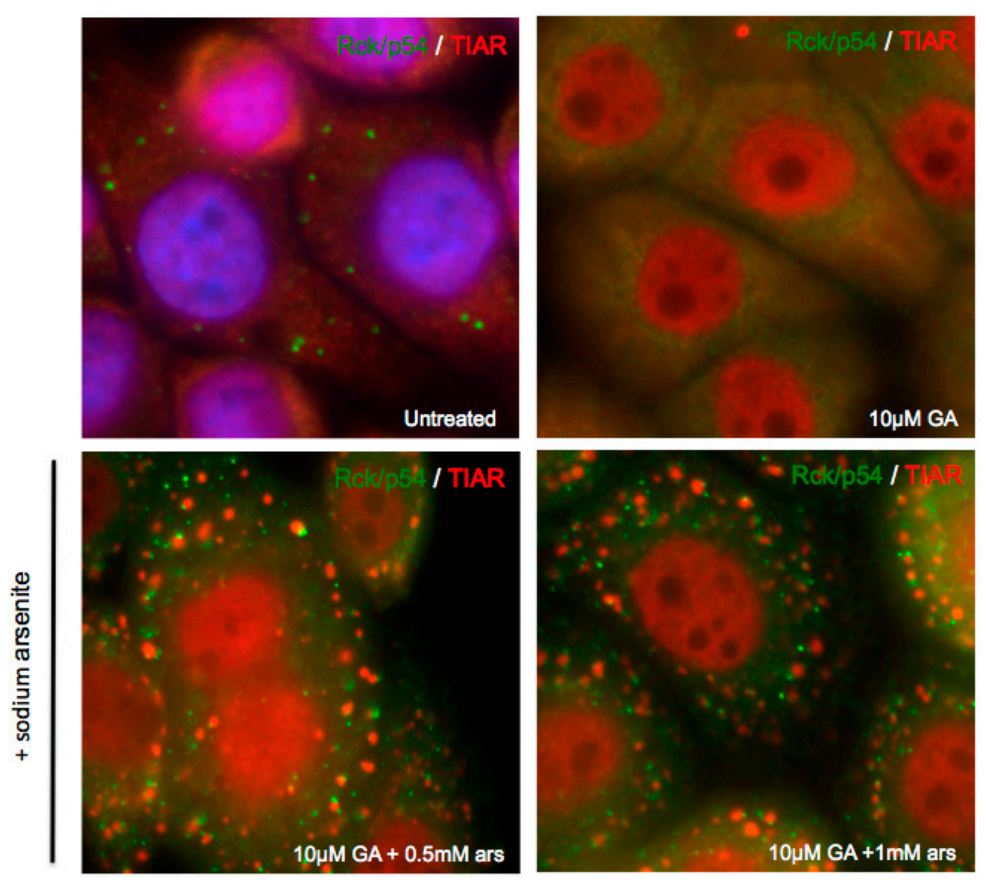

Figure 5. Exogenous stress restores chemically dispersed P-bodies. A549 cells were treated with $10 \mu \mathrm{M}$ geldanamycin (GA) for $16 \mathrm{~h}$ and subsequently fixed for immunofluorescence or treated with sodium arsenite for $30 \mathrm{~min}$ prior to fixation. Cells were then incubated with antibodies against PB marker Rck/p54 (green) and SG marker TIAR (red).
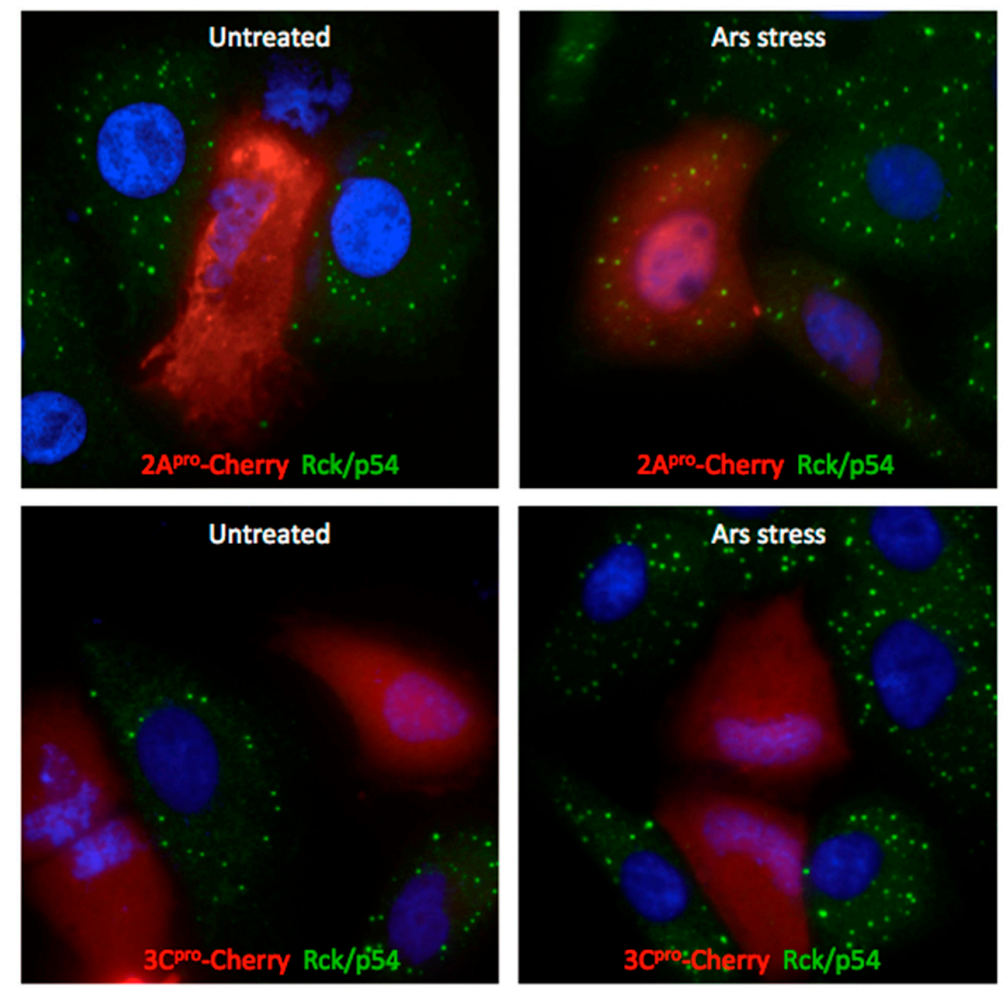

Figure 6. Exogenous stress rescues PBs disrupted by $2 \mathrm{~A}^{\text {pro }}$ but not $3 \mathrm{C}^{\text {pro }}$. A549 cells were

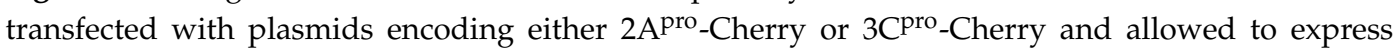
for $16 \mathrm{~h}$. Cells were then treated with 500-induced PB format for $30 \mathrm{~min}$, fixed, and processed for immunofluorescence. Cells were the incubated with anti-Rck/p54 antibody (green). 


\subsection{Exogenous Stress Rescues PBs Disrupted by PV 2A pro but not 3C pro}

Since both $2 \mathrm{~A}^{\text {pro }}$ and $3 \mathrm{C}^{\text {pro }}$ expression reduced the levels of endogenous $\mathrm{PBs}$ and we have no known targets of $2 \mathrm{~A}^{\text {pro }}$ that associate with $\mathrm{PBs}$, we explored if the proteases used a similar mechanism or targeted a common assembly pathway. We added sodium arsenite to A549 cells expressing either $2 \mathrm{~A}^{\text {pro }}$ or $3 \mathrm{C}^{\text {pro }}$ Cherry fusions in an attempt to restore $\mathrm{PB}$ formation similarly to when $\mathrm{PB}$ were chemically disrupted with GA. As previously demonstrated (Figure 4), expression of either 2A or 3C proteinase significantly disperses endogenous $\mathrm{PBs}$, as marked by Rck/p54. Following the addition of sodium arsenite, $\mathrm{PB}$ foci are rescued in cells expressing $2 \mathrm{~A}^{\text {pro }}$ (Figure 6). This suggests that $2 \mathrm{~A}^{\text {pro }}$ may cleave a target protein or inhibit a process involved in the maintenance or formation of constitutively expressed $\mathrm{PB}$, but one that is not essential for the aggregation of stress-induced PBs. In contrast, exogenous stress does not restore PB foci in cells expressing $3 \mathrm{C}^{\text {pro }}$ (Figure 6), suggesting that $3 \mathrm{C}^{\text {pro }}$ targeted protein(s) are essential for constitutive or stress-induced $\mathrm{PB}$ formation. These findings strengthen the hypothesis that PV 2A and $3 \mathrm{C}$ proteinases have cellular targets in different PB assembly pathways.
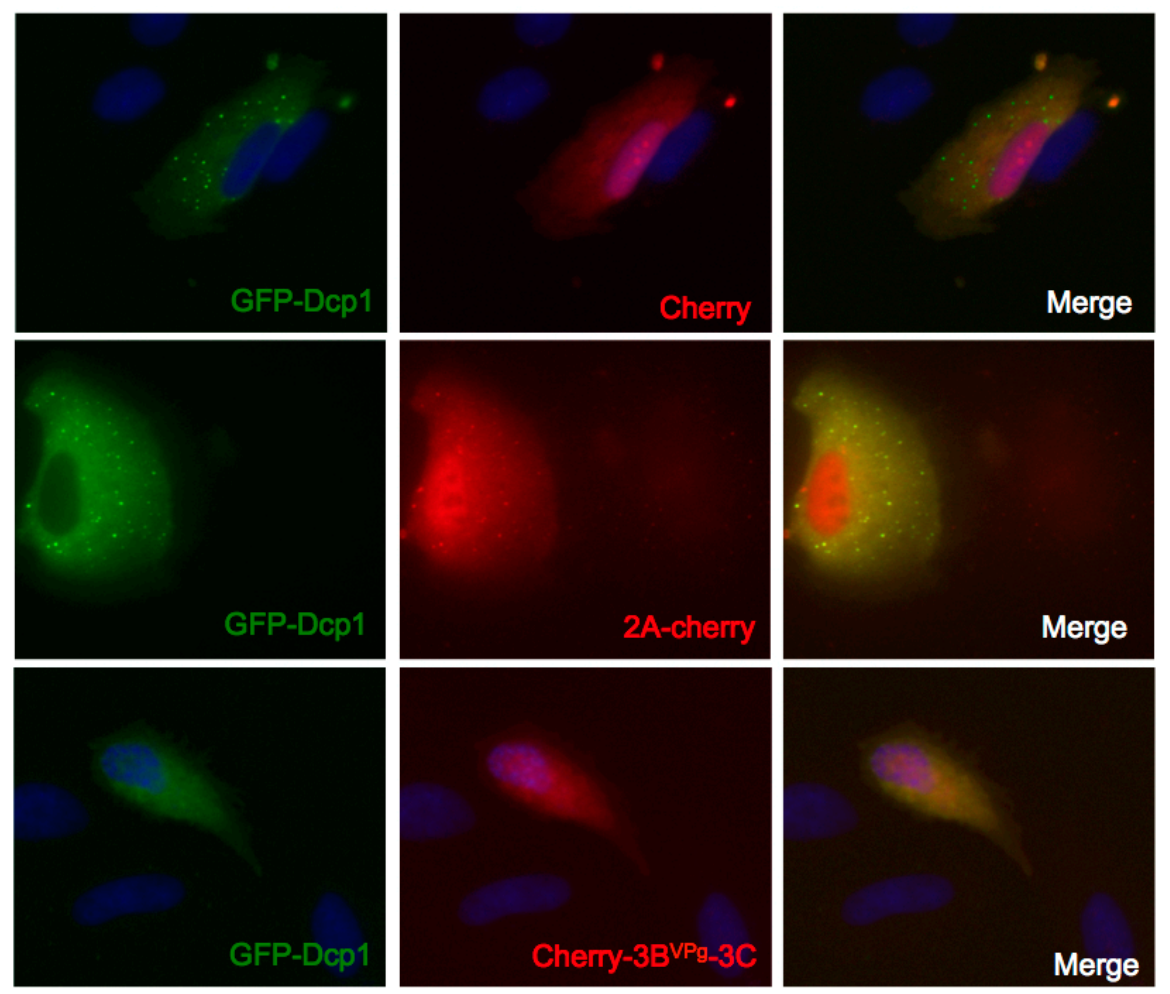

Figure 7. Expression of $3 C^{\text {pro }}$, but not $2 A^{\text {pro }}$, disrupts GFP-Dcp1a containing PB. A549 cells were co-transfected with expression vectors encoding GFP-Dcpla (green) and mCherry, 2A ${ }^{\text {pro }}$-Cherry, or Cherry-3BVPg-3Cpro (red). After $16 \mathrm{~h}$ of co-expression the cells were fixed and processed for immunofluorescence.

\subsection{Expression of $3 C^{\text {pro }}$, but not $2 A^{\text {pro }}$, Disrupts GFP-Dcp1a Containing PB}

The decapping complex protein Dcp1a is a PB component targeted by virus infection. Previous data demonstrated that ectopic expression of Dcp1a inhibits PV translation, and that this translation repression can be relieved by the inhibition of protein kinase $R$ (PKR) [25]. To investigate if PV proteases were capable of disrupting PBs containing ectopic GFP-Dcp1a, we co-expressed GFP-Dcp1a with plasmids encoding PV proteases. The expression of mCherry did not significantly alter the size or number of GFP-Dcp1a containing PBs in transfected cells, similar to the lack of Cherry-mediated

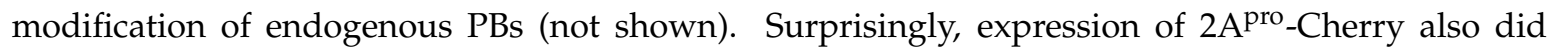


not significantly disperse GFP-Dcp1a containing PBs (Figure 7). This result is in contrast to what is observed with $2 A^{\text {pro }}$ expression when endogenous PBs are measured (Figure 4). Since $2 A^{\text {pro }}$ is not capable of cleaving Dcp1a, this may suggest that GFP-Dcp1a stabilizes or supplements PB assembly. In contrast, the expression of $3 C^{\text {pro }}$ significantly disperses GFP-Dcp1a containing PBs, similarly to what is observed during PV infection when PKR is inhibited allowing viral translation (Figure 7). Two forms of $3 C^{\text {pro }}$ were tested, one with $3 C^{\text {pro }}$ cloned upstream of mCherry and another cloned downstream of mCherry with a cleavable VPg-linker, which gave similar results; the latter is shown. This suggests that while GFP-Dcp1a expression may prevent $\mathrm{PB}$ disruption by $2 \mathrm{~A}^{\text {pro }}, 3 \mathrm{C}^{\text {pro }}$ is capable of cleaving GFP-Dcp1a, as well as endogenous Dcp1a, and other factors potentially required for PB formation. This further strengthens the idea that $2 \mathrm{~A}$ and $3 \mathrm{C}$ proteinases mediate the dispersal of PBs through differential mechanisms or targets.

\section{Discussion}

We have previously shown that PV blocks the formation of SGs and PBs during infection. In both cases, disruption of the RNA granules was linked to $3 C^{\text {pro }}$ and target cellular proteins were identified in SGs (G3BP1) and PBs (Dcp1a) that are substrates of $3 C^{\text {pro }}[13,14]$. Though cleavage of G3BP1 is important in the viral disruption of SGs, it is possible that viral manipulation of other proteins and pathways may contribute to SG inhibition. Likewise, the role of Dcpla cleavage in PB assembly/function has remained more enigmatic and we hypothesized that multiple viral proteins could repress PB formation. Thus, we conducted a screen of all PV gene products to determine their roles in inducing SGs or inhibiting SG and PB assembly or maintenance. Our results confirmed that PV 2A $A^{\text {pro }}$ was a strong inducer of SG assembly, similar to a previous report for CVB3 2A ${ }^{\text {pro }}$ [26] and that no other viral proteins induced SGs. In contrast, $2 \mathrm{~A}^{\text {pro }}$ displayed only weak activity towards inhibition of Ars-induced SGs. However, we noted inclusion of Cherry-2A ${ }^{\text {pro }}$ into some unique foci that contained the SG nucleating protein G3BP, but not Tia1, indicating these foci were not bona fide SGs. However, the colocalization of $2 \mathrm{~A}^{\text {pro }}$ and G3BP prompted a closer examination of the role of viral proteinases in cleavage of G3BP2. G3BP1 and G3BP2 have high homology, they interact with each other and newer reports suggest they carry out similar roles in supporting stress granule assembly $[18,19,27]$. Knockdown of either factor results in compensatory increased expression of the other form of G3BP in cells (not shown) and they both interact with factors ubiquitin specific peptidase 10 (USP10) and Caprin. We have not previously examined G3BP2 cleavage in infected cells as reagents were not available. G3BP2 cleavage did not occur during PV infection, and both $2 A$ and $3 C$ proteinases failed to cleave G3BP2 in vitro. Since cleavage of only G3BP1 results in such dramatic inhibition of SGs, this suggests human enteroviruses evolved to cleave the G3BP1 homolog that is functionally more important in SG assembly or innate immune signaling [28,29]. Conversely, it is possible that G3BP1 cleavage products are dominant negative inhibitors of SG assembly that can poison G3BP2-mediated assembly.

In our screen of viral factors that inhibit SG assembly, 3Cpro was the strongest repressor of SGs, in keeping with its role in cleaving G3BP1. Unlike 3C ${ }^{\text {pro }}$, the precursor 3CD, which contains proteolytic activity with altered cleavage specificity [20] had no effect on SG assembly. This suggests that G3BP1 is not cleaved by 3CD, though attempts to confirm this failed due to its toxicity and low expression level in cells. The other viral polypeptides had no strong effect on SGs, though we noted that P1 capsid proteins and 2A $\mathrm{A}^{\text {pro }}$ displayed a modest inhibitory effect on SG assembly but did not block them.

We report here the first screen of enteroviral proteins that inhibit PB formation. PB assembly is not well understood but requires influx of partially deadenylated mRNPs, may be organized spatially by Rck/p54 and association of key RNA-decay moieties, and seems to be transiently associated with mitochondria via dynamic microtubule networks, which seem to affect the assembly of active RNA-induced silencing complex (RISC) and Ago2 association with PBs [30-33]. Surprisingly, we found in this work that $2 \mathrm{~A}^{\text {pro }}$ blocked endogenous $\mathrm{PB}$ formation more effectively than $3 \mathrm{C}^{\text {pro }}$. No host 
targets of $2 \mathrm{~A}^{\text {pro }}$ were identified previously when a series of candidate PB factors were screened that could account for disruption of PBs. This included several mRNA decapping complex components, poly(A)-nucleases, [13]. A previous report indicated 2A $\mathrm{A}^{\text {pro }}$ cleaved Gemin 3 of the spliceosomal Sm core assembly machinery, but no other constituents [34]. These factors are not known to play roles in PBs, however Gemin 3 can complex with Ago2, a key PB component, thus an indirect role for Gemin 3 cleavage in $\mathrm{PB}$ assembly is possible. We also determined that expression of $3 \mathrm{CD}$ and even $3 \mathrm{D}^{\text {pol }}$ lowered average numbers of PBs in cells to about $50 \%$ normal levels. In the case of $3 C D$, this may be due to partial cleavage of Dcp1a or other factors. Future work will attempt to discover novel $2 \mathrm{~A}^{\text {pro }}, 3 \mathrm{C}^{\text {pro }}$ and $3 \mathrm{CD}$ cleavage targets that may affect $\mathrm{PB}$ biology.

Since there were no known cellular targets of $2 \mathrm{~A}^{\text {pro }}$ that are thought to play roles in $\mathrm{PB}$ biology, we tested pathways of $\mathrm{PB}$ assembly using inhibitors (geldanamycin) and promoters (stress) to probe for differences in the functional outcomes imposed by each proteinase. In the current understanding of the mRNA cycle among RNA granules, mRNA cargo may enter PBs directly via mRNP remodeling in conjunction with miRNA silencing and partial poly(A) shortening or by exchange of translationally-stalled mRNP cargo from SGs, which also must involve extensive mRNPs remodeling $[1,33,35]$. The precise mechanism of GA-inhibition of PBs is not known, but may involve dysfunction of Argonaut proteins or GW182 [22,36] involved in miRNA loading. GA mimics ATP-binding to Hsp90 leading to Hsp90-client proteins becoming unstable and degrading [37]. Thus, in unstressed cells GA may affect loading of mRNPs via the miRNA pathway primarily.

Another mechanism promoted by GA may involve functions of eIF4E and its transporter protein 4E-T. Although most translation initiation factors only enter SGs, eIF4E and 4E-T are exceptions also found in PBs [6,23]. We found that stress could override the blockage of constitutive PB assembly imposed by geldanamycin. In these cells the percentage of PBs found interacting with SGs was unusually high, indicating a defect in mechanisms that promote cargo exchange or separation of the RNA granules. Both $2 \mathrm{~A}$ and $3 \mathrm{C}$ proteinases blocked constitutive assembly of PBs, however arsenite stress rescued $\mathrm{PBs}$ in the presence of $2 \mathrm{~A}^{\text {pro }}$ but not $3 \mathrm{C}^{\text {pro }}$. This may suggest that $2 \mathrm{~A}^{\text {pro }}$ targets some components in the miRNA-Argonaut pathway of $\mathrm{PB}$ assembly, whereas $3 \mathrm{C}^{\text {pro }}$ targets a factor involved in the SG-mRNP cargo exchange pathway. Factors that promote SG-PB exchange are poorly defined, but may include tristetraprolin and BRF1 [1]. We note that both $2 \mathrm{~A}^{\text {pro }}$ and $3 \mathrm{C}^{\text {pro }}$ cleave poly(A)-binding protein (PABP), which is a major constituent of SGs but not PBs $[38,39]$. PABP regulates deadenylation of mRNPs by binding Pan3 and stimulating Pan2 nuclease activity and interacting with GW182 [33,40-42] through a C-terminal motif that is removed by both viral proteinases. Though they affect PABP similarly, they cleave different pools of PABP in the cell, with $3 C^{\text {pro }}$ primarily directed at translating polysome-associated PABP, whereas $2 \mathrm{~A}^{\text {pro }}$ cleaves PABP not associated with active translation [43].

Finally, it is known that the molecular constituents of PBs differ between unstressed and stressed cells; the PBs of the latter contain YB-1, and likely other novel factors [6]. Further, overexpression of Dcp1a forms larger PBs that are resistant to dissolution with emetine, unlike endogenous PBs. Also, we have shown that expression of Dcpla activates PKR and downstream stress pathways that block translation and will drive PB assembly [25]. Our experiments showing that only $3 C^{\text {pro }}$ could disrupt $\mathrm{PBs}$ with ectopic Dcp1a is consistent with $3 \mathrm{C}^{\text {pro }}$, not $2 \mathrm{~A}^{\text {pro }}$, dismantling the stress-driven pathway of PB assembly. Future work should determine the role of SG assembly itself in this pathway and to what extent PB assembly is driven by SGs under stress conditions.

Acknowledgments: This work was funded by NIH Public Health Service grants AI50237 (R.E.L.) and CA168872 and GM111700-01 (N.K.) and supported by the Integrated Microscopy Core at Baylor College of Medicine with funding from the NIH (HD007495, DK56338, and CA125123), the Dan L. Duncan Cancer Center, and the John S. Dunn Gulf Coast Consortium for Chemical Genomics. The authors kindly thank Todd R. Albrecht and Eric J. Wagner for providing modified Cherry expression plasmids.

Author Contributions: J.D. and R.L. conceived and designed the experiments; J.D., W.C.T., and R.L. performed the experiments; J.D. and R.L. analyzed the data; J.D. and R.L. wrote the paper. 
Conflicts of Interest: The authors declare no conflict of interest.

\section{References}

1. Kedersha, N.; Stoecklin, G.; Ayodele, M.; Yacono, P.; Lykke-Andersen, J.; Fritzler, M.J.; Scheuner, D.; Kaufman, R.J.; Golan, D.E.; Anderson, P. Stress granules and processing bodies are dynamically linked sites of mRNP remodeling. J. Cell Biol. 2005, 169, 871-884. [CrossRef] [PubMed]

2. Anderson, P.; Kedersha, N. RNA granules. J. Cell Biol. 2006, 172, 803-808. [CrossRef] [PubMed]

3. Kedersha, N.; Ivanov, P.; Anderson, P. Stress granules and cell signaling: More than just a passing phase? Trends Biochem. Sci. 2013, 38, 494-506. [CrossRef] [PubMed]

4. Teixeira, D.; Sheth, U.; Valencia-Sanchez, M.A.; Brengues, M.; Parker, R. Processing bodies require RNA for assembly and contain nontranslating mRNAs. RNA 2005, 11, 371-382. [CrossRef] [PubMed]

5. Eulalio, A.; Behm-Ansmant, I.; Izaurralde, E. P bodies: At the crossroads of post-transcriptional pathways. Nat. Rev. Mol. Cell Biol. 2007, 8, 9-22. [CrossRef] [PubMed]

6. Kedersha, N.; Anderson, P. Mammalian stress granules and processing bodies. Methods Enzymol. 2007, 431, 61-81. [PubMed]

7. Anderson, P.; Kedersha, N.; Ivanov, P. Stress granules, P-bodies and cancer. Biochim. Biophys. Acta 2015, 1849, 861-870. [CrossRef] [PubMed]

8. Weil, T.T.; Parton, R.M.; Herpers, B.; Soetaert, J.; Veenendaal, T.; Xanthakis, D.; Dobbie, I.M.; Halstead, J.M.; Hayashi, R.; Rabouille, C.; et al. Drosophila patterning is established by differential association of mRNAs with P bodies. Nat. Cell Biol. 2012, 14, 1305-1315. [CrossRef] [PubMed]

9. Wolozin, B. Physiological protein aggregation run amuck: Stress granules and the genesis of neurodegenerative disease. Discov. Med. 2014, 17, 47-52. [PubMed]

10. Buchan, J.R. mRNP granules. Assembly, function, and connections with disease. RNA Biol. 2014, 11, 1019-1030. [CrossRef] [PubMed]

11. Tsai, W.C.; Lloyd, R.E. Cytoplasmic RNA granules and viral infection. Annu. Rev. Virol. 2014, 1, 147-170. [CrossRef]

12. Reineke, L.C.; Lloyd, R.E. Diversion of stress granules and P-bodies during viral infection. Virology 2013, 436, 255-267. [CrossRef] [PubMed]

13. Dougherty, J.D.; White, J.P.; Lloyd, R.E. Poliovirus-mediated disruption of cytoplasmic processing bodies. J. Virol. 2011, 85, 64-75. [CrossRef] [PubMed]

14. White, J.P.; Cardenas, A.M.; Marissen, W.E.; Lloyd, R.E. Inhibition of cytoplasmic mRNA stress granule formation by a viral proteinase. Cell Host Microbe 2007, 2, 295-305. [CrossRef] [PubMed]

15. White, J.P.; Lloyd, R.E. Poliovirus unlinks TIA1 aggregation and mRNA stress granule formation. J. Virol. 2011, 85, 12442-12454. [CrossRef] [PubMed]

16. Lloyd, R.E.; Grubman, M.J.; Ehrenfeld, E. Relationship of p220 cleavage during picornavirus infection to 2A proteinase sequencing. J. Virol. 1988, 62, 4216-4223. [PubMed]

17. Mazroui, R.; Sukarieh, R.; Bordeleau, M.-E.; Kaufman, R.J.; Northcote, P.; Tanaka, J.; Gallouzi, I.; Pelletier, J. Inhibition of ribosome recruitment induces stress granule formation independently of eukaryotic initiation factor 2alpha phosphorylation. Mol. Biol. Cell 2006, 17, 4212-4219. [CrossRef] [PubMed]

18. Bidet, K.; Dadlani, D.; Garcia-Blanco, M.A. G3BP1, G3BP2 and CAPRIN1 are required for translation of interferon stimulated mRNAs and are targeted by a dengue virus non-coding RNA. PLoS Pathog. 2014, 10, e1004242. [CrossRef] [PubMed]

19. Matsuki, H.; Takahashi, M.; Higuchi, M.; Makokha, G.N.; Oie, M.; Fujii, M. Both G3BP1 and G3BP2 contribute to stress granule formation. Genes Cells 2013, 18, 135-146. [CrossRef] [PubMed]

20. Ypma-Wong, M.F.; Dewalt, P.G.; Johnson, V.H.; Lamb, J.G.; Semler, B.L. Protein 3CD is the major poliovirus proteinase responsible for cleavage of the P1 capsid precursor. Virology 1988, 166, 265-270. [CrossRef]

21. Blair, W.S.; Nguyen, J.H.; Parsley, T.B.; Semler, B.L. Mutations in the poliovirus 3CD proteinase S1-specificity pocket affect substrate recognition and RNA binding. Virology 1996, 218, 1-13. [CrossRef] [PubMed]

22. Johnston, M.; Geoffroy, M.-C.; Sobala, A.; Hay, R.; Hutvagner, G. HSP90 protein stabilizes unloaded argonaute complexes and microscopic P-bodies in human cells. Mol. Biol. Cell 2010, 21, 1462-1469. [CrossRef] [PubMed] 
23. Matsumoto, K.; Minami, M.; Shinozaki, F.; Suzuki, Y.; Abe, K.; Zenno, S.; Matsumoto, S.; Minami, Y. Hsp90 is involved in the formation of P-bodies and stress granules. Biochem. Biophys. Res. Commun. 2011, 407, 720-724. [CrossRef] [PubMed]

24. Suzuki, Y.; Minami, M.; Suzuki, M.; Abe, K.; Zenno, S.; Tsujimoto, M.; Matsumoto, K.; Minami, Y. The Hsp90 inhibitor geldanamycin abrogates colocalization of eIF4E and eIF4E-transporter into stress granules and association of eIF4E with eIF4G. J. Biol. Chem. 2009, 284, 35597-35604. [CrossRef] [PubMed]

25. Dougherty, J.D.; Reineke, L.C.; Lloyd, R.E. mRNA decapping enzyme 1a (Dcpla)-induced translational arrest through protein kinase $\mathrm{R}$ (PKR) activation requires the N-terminal enabled vasodilator-stimulated protein homology 1 (EVH1) domain. J. Biol. Chem. 2014, 289, 3936-3949. [CrossRef] [PubMed]

26. Wu, S.; Wang, Y.; Lin, L.; Si, X.; Wang, T.; Zhong, X.; Tong, L.; Luan, Y.; Chen, Y.; Li, X.; et al. Protease 2A induces stress granule formation during coxsackievirus B3 and enterovirus 71 infections. Virol. J. 2014, 11, 1-10. [CrossRef] [PubMed]

27. Kobayashi, T.; Winslow, S.; Sunesson, L.; Hellman, U.; Larsson, C. PKC $\alpha$ Binds G3BP2 and Regulates Stress Granule Formation Following Cellular Stress. PLoS ONE 2012, 7, e35820. [CrossRef] [PubMed]

28. Reineke, L.C.; Lloyd, R.E. The Stress Granule Protein G3BP1 Recruits Protein Kinase R to Promote Multiple Innate Immune Antiviral Responses. J. Virol. 2015, 89, 2575-2589. [CrossRef] [PubMed]

29. Reineke, L.C.; Kedersha, N.; Langereis, M.A.; van Kuppeveld, F.J.M.; Lloyd, R.E. Stress granules regulate double-stranded RNA-dependent protein kinase activation through a complex containing G3BP1 and Caprin1. MBio 2015, 6, e02486. [CrossRef] [PubMed]

30. Huang, L.; Mollet, S.; Souquere, S.; Le Roy, F.; Ernoult-Lange, M.; Pierron, G.; Dautry, F.; Weil, D. Mitochondria associate with P-bodies and modulate microRNA-mediated RNA interference. J. Biol. Chem. 2011, 286, 24219-24230. [CrossRef] [PubMed]

31. Ernoult-Lange, M.; Baconnais, S.; Harper, M.; Minshall, N.; Souquere, S.; Boudier, T.; Bénard, M.; Andrey, P.; Pierron, G.; Kress, M.; et al. Multiple binding of repressed mRNAs by the P-body protein Rck/p54. RNA 2012, 18, 1702-1715. [CrossRef] [PubMed]

32. Minshall, N.; Kress, M.; Weil, D.; Standart, N. Role of p54 RNA helicase activity and its C-terminal domain in translational repression, P-body localization and assembly. Mol. Biol. Cell 2009, 20, 2464-2472. [CrossRef] [PubMed]

33. Zheng, D.; Ezzeddine, N.; Chen, C.-Y.A.; Zhu, W.; He, X.; Shyu, A.-B. Deadenylation is prerequisite for P-body formation and mRNA decay in mammalian cells. J. Cell Biol. 2008, 182, 89-101. [CrossRef] [PubMed]

34. Almstead, L.L.; Sarnow, P. Inhibition of U snRNP assembly by a virus-encoded proteinase. Genes Dev. 2007, 21, 1086-1097. [CrossRef] [PubMed]

35. Parker, R.; Sheth, U. P bodies and the control of mRNA translation and degradation. Mol. Cell 2007, 25, 635-646. [CrossRef] [PubMed]

36. Pare, J.M.; Tahbaz, N.; López-Orozco, J.; LaPointe, P.; Lasko, P.; Hobman, T.C. Hsp90 regulates the function of argonaute 2 and its recruitment to stress granules and P-bodies. Mol. Biol. Cell 2009, 20, 3273-3284. [CrossRef] [PubMed]

37. Roe, S.M.; Prodromou, C.; O’Brien, R.; Ladbury, J.E.; Piper, P.W.; Pearl, L.H. Structural basis for inhibition of the Hsp90 molecular chaperone by the antitumor antibiotics radicicol and geldanamycin. J. Med. Chem. 1999, 42, 260-266. [CrossRef] [PubMed]

38. Joachims, M.; Van Breugel, P.C.; Lloyd, R.E. Cleavage of poly(A)-binding protein by enterovirus proteases concurrent with inhibition of translation in vitro. J. Virol. 1999, 73, 718-727. [PubMed]

39. Kuyumcu-Martinez, N.M.; Van Eden, M.E.; Younan, P.; Lloyd, R.E. Cleavage of poly(A)-binding protein by poliovirus 3C protease inhibits host cell translation: A novel mechanism for host translation shutoff. Mol. Cell. Biol. 2004, 24, 1779-1790. [CrossRef] [PubMed]

40. Fabian, M.R.; Cieplak, M.K.; Frank, F.; Morita, M.; Green, J.; Srikumar, T.; Nagar, B.; Yamamoto, T.; Raught, B.; Duchaine, T.F.; et al. miRNA-mediated deadenylation is orchestrated by GW182 through two conserved motifs that interact with CCR4-NOT. Nat. Struct. Mol. Biol. 2011, 18, 1211-1217. [CrossRef] [PubMed]

41. Fabian, M.R.; Mathonnet, G.; Sundermeier, T.; Mathys, H.; Zipprich, J.T.; Svitkin, Y.V.; Rivas, F.; Jinek, M.; Wohlschlegel, J.; Doudna, J.A.; et al. Mammalian miRNA RISC recruits CAF1 and PABP to affect PABP-dependent deadenylation. Mol. Cell 2009, 35, 868-880. [CrossRef] [PubMed] 
42. Funakoshi, Y.; Doi, Y.; Hosoda, N.; Uchida, N.; Osawa, M.; Shimada, I.; Tsujimoto, M.; Suzuki, T.; Katada, T.; Hoshino, S.-I. Mechanism of mRNA deadenylation: Evidence for a molecular interplay between translation termination factor eRF3 and mRNA deadenylases. Genes Dev. 2007, 21, 3135-3148. [CrossRef] [PubMed]

43. Rivera, C.I.; Lloyd, R.E. Modulation of enteroviral proteinase cleavage of poly(A)-binding protein (PABP) by conformation and PABP-associated factors. Virology 2008, 375, 59-72. [CrossRef] [PubMed]

(C) 2015 by the authors; licensee MDPI, Basel, Switzerland. This article is an open access article distributed under the terms and conditions of the Creative Commons by Attribution (CC-BY) license (http:/ / creativecommons.org/licenses/by/4.0/). 